\title{
Urban Road Network Extraction Based on Fuzzy ART, Radon Transform and Morphological Operations on DSM Data
}

\author{
Darlis Herumurti ${ }^{1}$, Keiichi Uchimura ${ }^{1}$ Gou Koutaki ${ }^{1}$, and Takumi Uemura ${ }^{2}$ \\ 1 Kumamoto University, 2-39-1, Kurokami, Chuo-ku, Kumamoto 860-8555, Japan \\ darlis@navi.cs.kumamoto-u.ac.jp \\ 2 Sojo University, 4-22-1, Ikeda, Chuo-ku, Kumamoto, 860-0082, Japan
}

\begin{abstract}
In urban areas, the main disadvantage of an aerial photo for road extraction is the shadow cast by buildings and the complexity of the road network. For this case, we used Digital Surface Model (DSM) data, which are based on the elevation of land surfaces. However, one of the problems associated with DSM data is the non-road area with the same road elevations, like parking places, parks, empty ground and so on. In this paper, we propose the Mixed ART clustering on histogram followed by region growing to extract the initial road and perform the road filter by opening operation with a line shape structuring element, where the line orientation is obtained from the Radon Transform. Finally, the road networks are constructed based on B-Spline curve from the skeleton of the extracted road. The experimental result shows that the proposed method improved the quality and the accuracy average within an acceptable time.
\end{abstract}

Keywords: road extraction, ART clustering, region growing, Radon transform, morphological operations.

\section{Introduction}

Recently, researches in Intelligent Transportation Systems (ITS) have contributed to and made a great impact on the modern social environment. Automatic road extraction is one interesting topic in this field, where it plays an important role in transportation planning and spatial development, particularly in urban areas, which are very challenging, as there are complex road networks and many buildings that cause shadow problems. The Digital Surface Model which is based on the elevation of land surfaces, including buildings, overcomes these problems. However, the drawback of this data is the difficulty of recognizing the non-road areas that have a similar elevation with the road and located near the road, like parking places, empty grounds, parks and so on.

Several studies have introduced this issue, and each method has its own advantages. Couloigner et al. propose an iterative and localized Radon Transform to detect the center-line from classified imagery [1. From the spatial clustering result, the input space is then partitioned into road component images, which 
is a subset of images. An iterative Radon Transform is applied locally to each road component image to detect road center-line segments based on an estimation of the line parameters and line widths. On the other hand, Maurya et al. employed the K-Mean as the road segmentation and morphological operation to remove the non-road areas such as building, parking lots and other similar objects [2]. Herumurti et al. proposed the method based on seeded region growing and Mixed ART [3]. First, data is segmented using seeded region growing, where the homogeneity measurement based on Mixed ART and the initial road is selected based on the highest area. Furthermore, the road merging is performed based on a match function in ART approach. However, it takes a longer running time. Another segmentation approach is proposed by Abbas Cheddada et al. by utilizing the histogram information [4. Abbas treated the histogram information as points geometry that could be separated by the voronoi diagram (VD) based on a delaunay triangulation (DT). This technique gave an efficient processing time.

Generally, a line detection approach is used in order to extract the road, and utilize the Hough Transform for this issue. The Hough Transform assesses the geometry information where a line segment is defined in terms of its polar coordinate parametric notation [5]. In at similar way, the Radon Transform has the ability to transform an image with lines into a domain of possible line parameters. The advantages of the Radon Transform technique are its ability to extract lines from very noisy images and its computational efficiency [1].

In this work, we proposed a method to perform a road network extraction based on image thresholding, Radon Transform, and morphological operations. The thresholding method was an appropriate technique because the elevation between the road and the buildings was significantly different. In addition, it ran extremely quick. The thresholding was done by selecting the threshold values from the ART clustering on a geometrical point histogram, followed by seeded region growing, producing the initial road and non-road. Morphological operations were then carried out on the initial road in order to smooth the road region and remove large areas. Furthermore, we performed the road-line filter based on an opening operation with the line structuring element, where the orientation of the line detection is based on the Radon Transform. Finally, the road networks were constructed using the B-spline curve from the skeleton of the extracted road.

Section 2 describes the materials and the methods we use to extract the initial road, remove the false alarm based on road line filtering and construct the road networks based on B-spline. Section 3 describes the experimental result on road extraction by comparing it to other methods. Finally, in section 4 , we conclude our work and discuss the limitations for future works.

\section{Materials and Methods}

This section describes the DSM data and the methods that are used in the experiment. The main objective of this research is to propose an automated urban road 
network extraction on DSM data based-on image thresholding by mixed ART clustering, region growing, and image morphology operations combining Radon Transform with morphological operations as the road line filter and construct the road network using the B-spline curve from the skeleton of the extracted road.

\subsection{Data Description}

In this paper, we used Sapporo and Shinjuku DSM data. Sapporo and Shinjuku are skyscraper districts in Japan that contain a lot of high-rise buildings. In addition, this area contains many occlusions and sloping roads. Table 1 shows the specification of the DSM data. The high-resolution data were taken in 2002 . Figure 1 shows Sapporo and its ground truth. Figure [1] (a) is the DSM data in the grayscale image visualization. Unlike in the aerial photo image, the DSM data are based on the elevation of land surface that do not contain shadows. Therefore, we expect to achieve a better result by using it. Figure [1](b)] is the digital ortho-aerial photography from Aero Asahi Corporation that corresponds to the DSM data. The aerial image data contain many building shadows that will disrupt the road extraction process. Figure [1](c) is binary images of the true model created manually from an existing digital road map. The road from this image is presented in black. We evaluate the result of road extraction based on this true model.

\subsection{Methods}

This section describes our proposed for the road extraction method called ARMOR, which stands for ART clustering, region growing, morphological operations and Radon Transform. Algorithm 1 shows the steps for road extraction. The input is from DSM data and the output is the extracted road and non-road.

\subsection{Preprocessing Based on Median Filter and CLAHE}

The pre-processing steps include median filtering and transforming the DSM to grayscale image. The grayscale image is only for the CLAHE and the tresholding to produce the initial road. In the region growing method, we used the DSM values directly. Median filtering is used to remove the noise without reducing the sharpness of the image. The second step is a contrast enhancement processing

Table 1. Data Specification

\begin{tabular}{|l|l|}
\hline Year of acquisition & 2002 \\
\hline Horizontal Error & $\pm 0.5 \mathrm{~m}$ \\
\hline Elevation Error & $\pm 0.15 \mathrm{~m}$ \\
\hline Resolution & $\pm 1.0 \mathrm{~m} /$ pixel \\
\hline
\end{tabular}



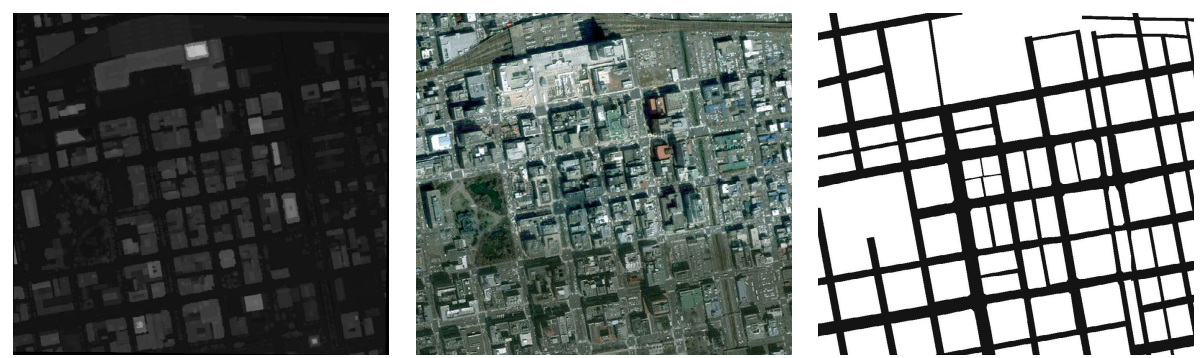

(a) DSM of Sapporo in (b) Aerial photo of Sapporo

(c) True model of Sapporo grayscale
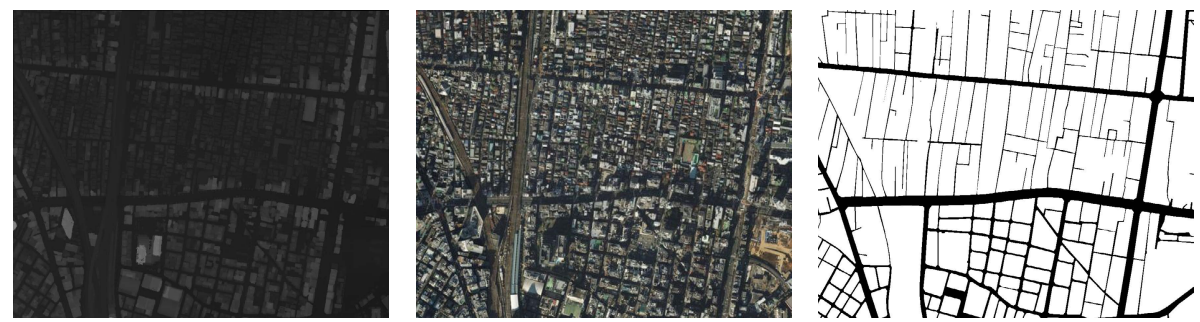

(d) DSM of Shinjuku in (e) Aerial photo of Shinjuku (f) True model of Shinjuku grayscale

Fig. 1. DSM data, RGB aerial photo and true model of Sapporo

based-on adaptive histogram equalization (CLAHE) instead of conventional histogram equalization (histeq), because the CLAHE produces an evenly distributed output value throughout the range [6].

\subsection{Thresholding by Mixed ART on Histogram}

To determine the threshold value, we employed the histogram analysis and performed the ART clustering. Furthermore, the cluster merging is performed to obtain the initial road based on ART measurement.

In Fuzzy ART, there are three important functions: the activation function, the match function, and the update function. On the other hand, the Symmetric Fuzzy ART (S Fuzzy ART) that is proposed by Baraldi and Ethem, adopts symmetric activation and match function. Therefore, S Fuzzy ART is more efficient because no search is needed as in the Fuzzy ART method [8]. In order to work with these functions, the data $(z)$ are scaled between 0 and 1 and to be normalized using complement coding $\left(z^{c}\right)$. For two dimensions data $z=\left(z_{1}, z_{2}\right)$, the input vector $(x)$ is defined by:

$$
x=\left(z, z^{c}\right)=\left(z_{1}, z_{2}, 1-z_{1}, 1-z_{2}\right)
$$




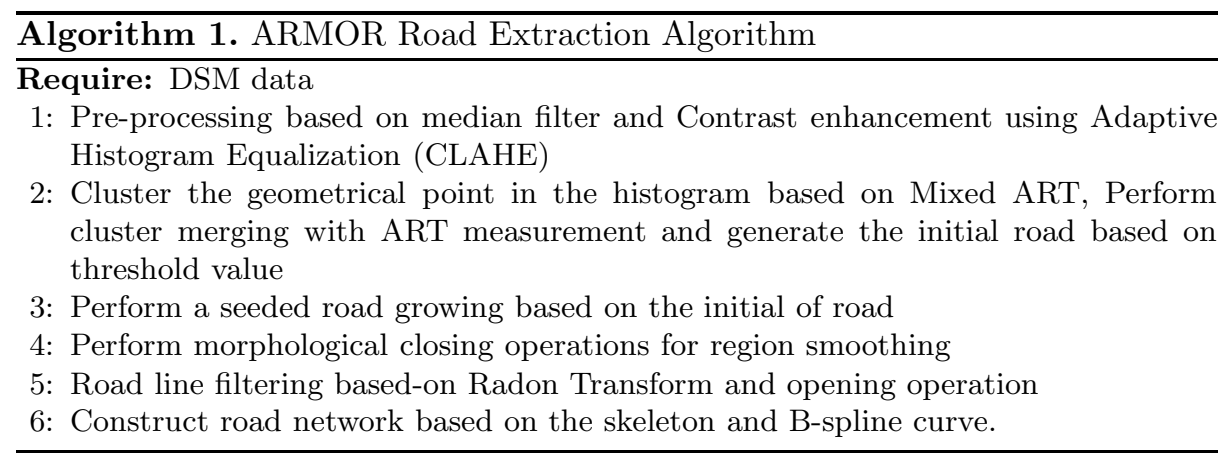

In this two dimensional feature space, each cluster $w_{j}$ is represented as a rectangle. This rectangle is defined by the cluster weight $w_{j}\left(\bigvee_{j} x,\left(\bigwedge_{j} x\right)^{c}\right)$ that store the information of bottom left and top right position of the rectangle. The following match function $M\left(x, w_{j}\right)$ is a mix of the match function from Fuzzy ART and Symmetric Fuzzy ART and measures the closeness between the input vector and a cluster weight $w_{j}$. The "resonance" occurs when $x$ is matched close enough with $\left(w_{j}\right)$. This closeness is measured by comparing the match value with the vigilance parameter $\rho: 0<\rho<1$, where $M\left(x, w_{j}\right)>\rho$ means a good match (resonance). [7.

$$
M\left(x, w_{j}\right)=\left(0.5 \times \frac{\left\|x \wedge w_{j}\right\|}{\|x\|}\right)+\left(0.5 \times \frac{1}{1+\|x-w\|}\right)
$$

If resonance occurs, then update the cluster weight with the update function:

$$
U\left(x, w_{j}\right)=x \wedge w_{j}
$$

Where the operator $\wedge$ in equations (2) and (3) is fuzzy AND operator:

$$
a \wedge b=\left(\min \left(a_{1}, b_{1}\right), \min \left(a_{2}, b_{2}\right)\right)
$$

And norm operator $\|$.$\| is defined by:$

$$
\|p\|=\sum_{i=1}^{D}|p|
$$

where $D$ is the feature length of the input vector $p$.

The complete Mixed ART algorithm can be seen in 3]. Figure 2](a)] shows the Mixed ART clustering based on geometrical points in the histogram. The geometrical points indicate the intensity of the histogram after performing the adaptive histogram equalization CLAHE. The cluster merging is done by selecting the cluster with the highest frequency and then merge the other cluster 
based on equation (2). The cluster merging result is shown with two small shade rectangles in Fig. 2|(a) Furthermore, the threshold values are selected based on the position (most left and most right) of the cluster after the merging, as shown in the big shaded rectangle. The initial road of Sapporo and Shinjuku resulting from the thresholding are shown in Figs. 2[(b) and 2[(d)

\subsection{Seeded Region Growing}

To expand the candidate road that resulted in the previous step, we employ the seed region-growing approach. In this paper, the seed points come from the perimeter of the candidate road. The road is then grown from these seed points to the adjacent based on the threshold value of the DSM Data. In the experiment, we used the threshold value of no more than $0.15 \mathrm{~m}$. This process was performed iteratively until all the seed data had been processed.

Equation (6) shows the homogeneity measurement $H(r)$ between the road $(r)$ and the adjacent. The homogeneity is defined by the difference between the current value $\left(z_{i}\right)$ and the adjacent $\left(z_{i+1}\right)$. If the difference of the current value and the adjacent is less than the threshold $(T)$, then the adjacent is the road $(r)$. Figures [2](c) and [2) (e) show the region growing results.

$$
H(i)=\text { True }: \text { if }\left|z_{i}-z_{i+1}\right|<T
$$

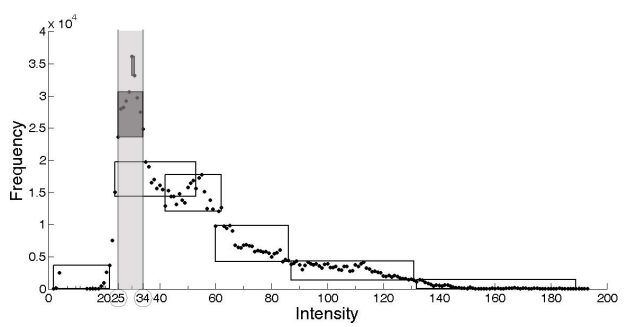

(a) Mixed ART clustering, cluster merging and threshold value selection

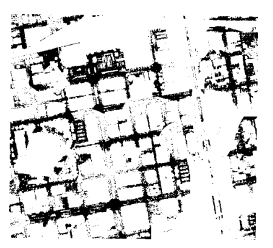

(b) Thresholding on Sapporo

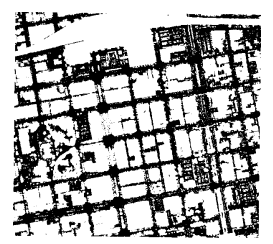

(c) Region growing on Sapporo

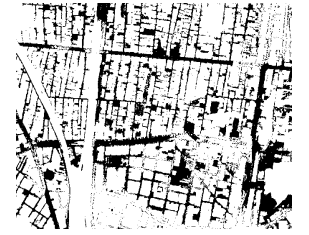

(d) Thresholding on Shinjuku

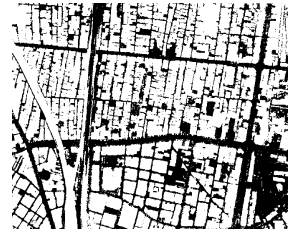

(e) Region growing on Shinjuku

Fig. 2. Mixed ART Clustering, thresholding and region growing 


\subsection{Region Smoothing Based-On Morphological Operations}

In this step, we performed a closing operation on the initial road to smooth the contour and fill small holes. We used a disk-shaped structuring element for the operation. To detect the large region, we performed an erode operation on the initial road (seen in black). Thus, a large area was detected. These large areas were then subtracted from the road. We used a rectangle-shaped structuring element for this work.

\subsection{Road-Line Detector Based-On Radon Transform and Morphological Operations}

In this step, we used a road line filter to minimize the false alarm by performing an opening operation with a line-shaped structuring element. To detect the line orientation, we performed the Radon Transform. The Radon Transform is the projection of the image intensity along any angle $\theta$ as defined in equation (7) and the locations of straight line in the image can be found by finding the locations of strong peaks in the Radon Transform matrix.

$$
R_{\theta}\left(x^{\prime}\right)=\int_{-\infty}^{+\infty} f\left(x^{\prime} \cos \theta-y^{\prime} \sin \theta, \quad x^{\prime} \sin \theta+y^{\prime} \cos \theta\right) \mathrm{d} y^{\prime}
$$

Figure 3 shows the illustration of road line-filtering on a synthetic road image. The synthetic data contain four road lines with some non-road areas. Figure [3) shows the Radon Transform of the synthetic image in Fig. 3i(a). The peaks of Radon Transform are shown in bright intensity. These peaks represented the lines in the image. The regional maximums of Radon Transform were then selected as shown in Fig. 3.(c), and we could obtain the dominant orientation angle that presented the lines. These orientation angles were used in the opening operation with a line-shaped structuring element to filter the road. Figures [i(d) and $3(\mathrm{e})$ show the opening operation result based on the selected orientation angle from the Radon Transform. This road filter could extract the road line on the specific angle and remove non-road areas. Combining all the filtering results, could extract road lines correctly as shown in Fig. [3] (f)

\subsection{Road Network Extraction Based on B-spline Curve}

In this step, we performed a morphology thin operation to produce the skeleton lines. The lines were then smoothed based on B-spline curve. We identified an end/joint pixel as a vertex in the skeleton where a joint pixel is defined by an interest pixel which has more than two neighbours in 8-connectivity, and a line between two adjacent vertices was then extracted. Furthermore, an extracted line was then smoothed based-on the B-spline. Figure L(b) and E(d) shows the B-spline curve in yellow, which represents the road-line network. 


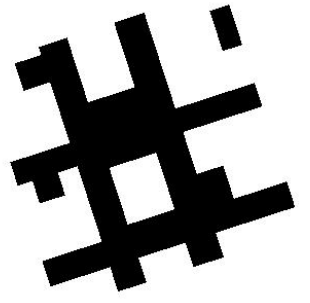

(a) synthetic road image

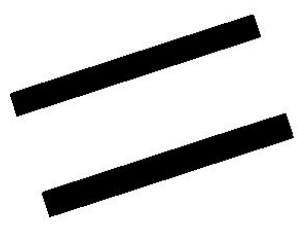

(d) Road filter $\theta=18^{\circ}$

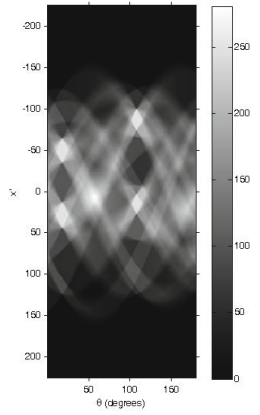

(b) Radon Transform

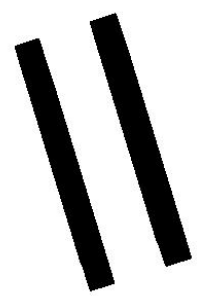

(e) Road filter $\theta=107^{\circ}$

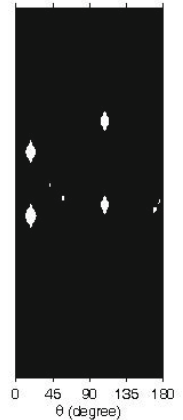

(c) Regional peaks

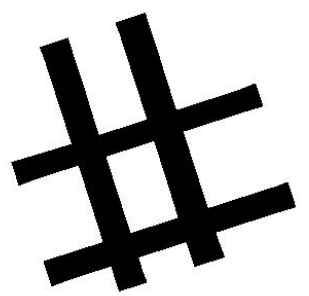

(f) Combining all peaks theta

Fig. 3. Road line filter based-on Radon Transform and opening operation on synthetic road image

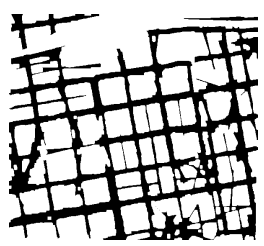

(a) Extracted road on Sapporo

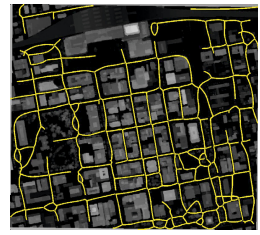

(b) Road network on Sapporo

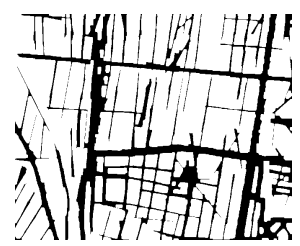

(c) Extracted road on Shinjuku

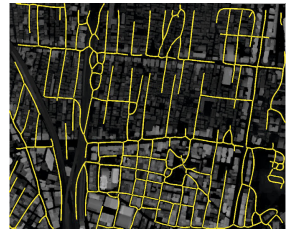

(d) Road network on Shinjuku

Fig. 4. Final road network extraction

\section{Experimental Result}

In this experiment, we used a desktop computer with a Pentium Core i5-2310M $2.9 \mathrm{GHz} \mathrm{CPU}$ and $4 \mathrm{~GB}$ memory. For software development, we used Matlab 2011b. The evaluation of each method was performed and measured by the 
Table 2. The evaluation of road extraction result

\begin{tabular}{|l|c|c|c|c|c|}
\hline Method & Quality & Eroad & Enon & Emean & Time(sec) \\
\hline ARMOR (proposed) & 0.51 & 0.74 & 0.86 & 0.80 & 2.86 \\
\hline Seed RG Mixed ART & 0.42 & 0.69 & 0.79 & 0.75 & 5.66 \\
\hline RG & 0.40 & 0.76 & 0.74 & 0.75 & 10.50 \\
\hline VD & 0.39 & 0.88 & 0.62 & 0.75 & 1.70 \\
\hline Mean Shift & 0.37 & 0.76 & 0.68 & 0.72 & 2.15 \\
\hline RGB aerial & 0.12 & 0.44 & 0.67 & 0.56 & 13.1 \\
\hline
\end{tabular}

quality and the accuracy of the road extraction. We used the evaluation criterion proposed by Heipke et al. 9]. The evaluation method uses True Positive $(T P)$, True Negative $(T N)$, False Positive $(F P)$ and False Negative $(F N)$ based on the true model that is shown in Fig. [1 $(c)$. $T P$ means the extracted area is the road, and $T N$ means the non-extracted area is a non-road. FP means a non-road is extracted as a road and $F N$ means an actual road is not extracted. Equations (8)-(11) shows the evaluation measurement.

$$
\begin{aligned}
& Q U A L I T Y=\frac{T P}{T P+F N+F P} \\
& E_{\text {road }}=\frac{T P}{T P+F N} \\
& E_{\text {non }}=\frac{T N}{T N+F P} \\
& E_{\text {mean }}=\frac{E_{\text {road }}+E_{\text {non }}}{2}
\end{aligned}
$$

Table 2 shows the evaluation result of the extracted road on Sapporo and Shinjuku DSM data. The proposed method, ARMOR, is compared to other methods such as, seeded region growing with Mixed ART (seed RG Mixed ART), Region Growing (RG), Mean-shift and Voronoi Diagram segmentation (VD) on the DSM data. In addition, we compared a road extraction result from the RGB aerial image using seeded region growing method.

\section{Conclusion}

In this work, we developed a method to extract the road from DSM data in an urban area. We addressed this problem by performing image thresholding, 
and region growing in order to obtain the initial road. Furthermore, the road filter is used on the initial road by utilizing the Radon Transform and followed by the opening operation with a line-shaped structuring element to remove a false alarm. Based on the experimental result, the proposed method, ARMOR, achieved the best quality and average of accuracy with $51 \%$ and $80 \%$ respectively.

Although the road-line filter can remove some of the non-roads from an extracted road, there is difficulty in recognizing the curved linear roads and narrow roads, where the extracted roads is tending toward linear roads. The computation time was not the quickest, yet it was acceptable. The future works of this research concern increasing the quality of the extracted road networks by recognizing the narrow and curved roads with a good performance.

\section{References}

1. Couloigner, I., Zhang, Q.: Iterative and Localized Radon Transform for Road Centerline Detection from Classified Imagery. In: Kamel, M.S., Campilho, A. (eds.) ICIAR 2007. LNCS, vol. 4633, pp. 1093-1104. Springer, Heidelberg (2007)

2. Maurya, R., Shalini, S., Gupta, P.R., Manis, K.S.: Road extraction using k-means clustering and morphological operations. International Journal of Advanced Engineering Sciences and Technologies 5(2), 290-295 (2011)

3. Herumurti, D., Uchimura, K., Koutaki, G., Uemura, T.: Automatic Road Extraction Using Seeded Region Growing with Mixed ART Method for DSM Data. IEE J. Transaction on Electronics, Information and Systems 133(1), 150-158 (2013)

4. Abbas, C., Dzulkifli, M., Azizah, A.M.: Exploiting Voronoi diagram properties in face segmentation and feature extraction. Elsevier Journal on Pattern Recognition 41(12), 3842-3859 (2008)

5. Duda, R.O., Hart, P.E.: Use of the Hough Transformation to Detect Lines and Curves in Pictures. Comm. ACM 15, 11-15 (1972)

6. Zuiderveld, K.: Contrast Limited Adaptive Histograph Equalization. In: Graphic Gems IV, pp. 474-485. Academic Press Professional, San Diego (1994)

7. Carpenter, G., Grossberg, S., Rosen, D.: Fuzzy ART Fast Stable Learning and Categorization of Analog Patterns by an Adaptive Resonance System. Elsevier Journal on Neural Network 4(6), 759-771 (1991)

8. Baraldi, A., Alpaydin, E.: Constructive feedforward art clustering networks-part i and ii. IEEE Trans. Neural Network 13, 645-677 (2002)

9. Heipke, C., Mayer, H., Wiedemann, C., Jamet, O.: Evaluation of Automatic Road Extraction. International Archives of Photogrammetry and Remote Sensing 32(3-2W3), 47-56 (1997) 